\title{
AN APPROACH FOR TOPOLOGY OPTIMIZATION-DRIVEN DESIGN FOR ADDITIVE MANUFACTURING
}

\author{
A. Nordin $ه$ \\ Lund University, Sweden \\ $\square$ axel.nordin@design.lth.se
}

\begin{abstract}
This paper describes an approach for designing lightweight components produced through additive manufacturing (AM). Lightweight design is often done through topology optimization (TO). However, the process of manually interpreting mesh-based and imprecise results from a TO into a geometry that fulfils all requirements is complex. To aid in this process, this paper suggest an approach based on combining overhang-constrained TO with lattice-based TO to automate complex tasks, retain parametric control, and to minimize manufacturing cost. The approach is validated through a benchmark part.
\end{abstract}

Keywords: additive manufacturing, optimisation, computational design methods, lightweight design

\section{Introduction}

This paper describes an approach for designing lightweight structural components, intended for production through additive manufacturing (AM). AM offers several benefits compared to conventional manufacturing, such as enabling geometrically complex parts to be manufactured with no added cost compared to simpler geometries. One of the major industrial applications of AM is the production of highly complex lightweight designs. The design of these parts can be based on experience or on the results from computer-aided simulation tools, with topology optimization being one of the most frequently used methods. Topology optimization enables the minimization or redistribution of material within a design space, given certain objectives and constraints. The typical workflow of performing a design based on topology optimization is to:

1. Establish the boundary conditions, for instance forces and supports

2. Establish the design space, that is, the volume within which the material can be placed

3. Define objectives and constraints for the optimization, for instance to minimize compliance and reduce the volume by $50 \%$

4. Perform the topology optimization

5. Interpret the results to produce a fully functional part

6. Validate the design

Most commercial computer-aided design (CAD) and analysis software include topology optimization functionality to some degree. Recently, the software functionality has been broadened to take into account manufacturability aspects, such as overhang angles for AM, and lattice-based structural optimization. However, the process of interpreting the result from the topology optimization, which is typically mesh-based and often quite imprecise, into a geometry that can be 
manufactured and fulfils all requirements can be time-consuming and complex. Although this poses a major obstacle in the industrial use of topology optimization for AM, it remains an open problem how to solve it (Liu et al., 2018).

\subsection{Aim}

To aid in the largely manual process of interpreting topology optimization results, this paper suggest an approach based on combining overhang-constrained topology optimization with lattice-based optimization. The design process is centred on three main aspects: automation of complex geometrical tasks, parametric controllability of the overall design, and minimizing manufacturing cost (e.g., minimize need for support material, minimize post-processing, and minimize build height). While the individual approaches of topology optimization and lattice size optimization have been the focus of many previous studies, this paper combines the approaches and validates the entire workflow to ensure its industrial usefulness.

\subsection{Structure of this paper}

This paper is organized as follows: In Section 2, the background to topology optimization within AM is given. In Section 3, the approach, a benchmark part, material and production system are described. In Section 4, the design process is described. In Section 5, the validation process is described. Finally, in Section 6, the concluding remarks are given.

\section{Background}

Topology optimization started gaining popularity in the late 1980s with important work being done by for instance Bendsøe and Kikuchi (1988). The technology has since then matured and has found many applications (Bendsøe and Sigmund, 2004; Sigmund and Maute, 2013) The most common use of topology optimization is to distribute material within a design space to maximize or minimize certain objectives. Topology optimization applied to parts intended for AM has been investigated by several publications, such as biomedical (Wang et al., 2016) and minimization of post-processing (Gaynor and Guest, 2016; Langelaar, 2016; Mirzendehdel and Suresh, 2016; Zegard and Paulino, 2016).

However, even if the topology optimization algorithm is adapted to AM, some degree of manual interpretation is usually needed to make the results from the optimization feasible for production and end-use. To accomplish this, there are several strategies, such as mesh smoothing algorithms available in software like Materialise Magics (Materialise, n.d.) or Autodesk Meshmixer ("Autodesk Meshmixer", n.d.) or poly-NURBS approaches available in for instance Altair Inspire (Altair, n.d.). Additionally, there are approaches based on using lattice structures with varying density to optimize the material usage, as can be found in, for instance, ANSYS Workbench (ANSYS, n.d.). However, all approaches either generate geometry that is hard to control parametrically, or requires a substantial amount of design work to create.

\subsection{Related works}

Liu and Ma (2016) provide a review of manufacturing oriented topology optimization methods. They bring up a number of promising approaches for generating parametrically modifiable optimization results, such as B-spline finite cell method combined with the level set function (Cai et al., 2014), isogeometric topology optimization (Seo et al., 2010), and translation of bitmap-based topology optimization results into an IGES format (Chacón et al., 2014). However, as Liu and Ma (2016) note, these approached are still experimental and generates geometries that are not friendly to model manipulation and manufacturing.

Bracket et al. (2011) identify the practical difficulties in converting the topology optimization result into a usable CAD model. They note that "Due to the high degree of topological complexity when optimizing for $\mathrm{AM}$, manual conversion to $\mathrm{CAD}$ is unreasonable, and current automatic methods of conversion have not been designed to handle this level of complexity." (Brackett et al., 2011, p. 355). Bracket et al. (2011) propose a mesh-based workflow, where modifications to the mesh resulting from the topology optimization are done by Boolean operations and a remeshing scheme is employed to improve the mesh 
quality for FEA. They also propose a workflow for mapping boundary conditions from CAD to the remeshed FE model, but provide no details on its implementation or efficacy.

Liu et al. (2018, p. 2475) note in their review of current and future trends in topology optimization for AM that "In the case that further editing of the topology design is needed, parameterization and creation of feasible CAD models are necessary, and tremendous research efforts have been spent attempting to address this issue" and that it remains an open problem.

\section{Method}

\subsection{Outline of the approach}

The design process is centred on three main aspects: automation of complex geometrical tasks, parametric controllability of the overall design, and minimizing manufacturing cost (e.g., minimize need for support material, minimize post-processing, and minimize build height). This is achieved through a process with two optimization steps: 1) optimize the topology of the structure to find a compromise between the stiffness of the structure, build height and the amount of support material needed; 2) create a simplified design based on the results; 3 ) perform a lattice-based topology optimization.

Automated lattice-based topology optimization is used to avoid manually creating the complex internal geometry. The exterior shell is based on the initial topology optimization but is left as simple as possible, while avoiding support material, to enable parametric control. The workflow is shown in Figure 1.

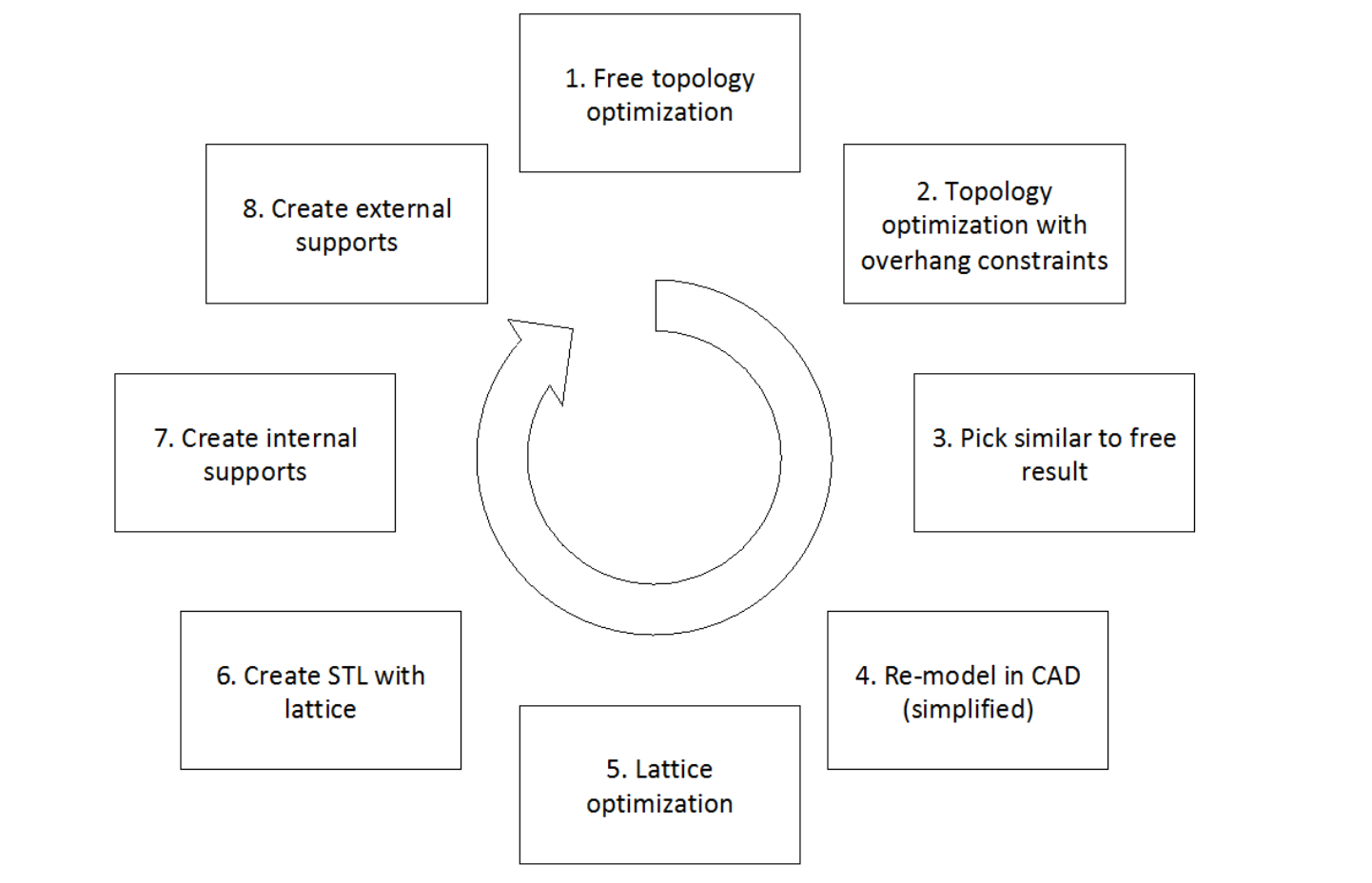

Figure 1. Diagram of the design workflow

The built-in topology optimization functionality of ANSYS Workbench (ANSYS Inc., n.d.) was used for all optimizations, although other software with overhang-constrained and lattice-based optimization could have been used.

Once the final design has been completed and support structures generated, the design is validated using simulation tools for structural analysis and build process simulation.

\subsection{Demonstrator part}

To demonstrate the approach proposed in this paper, the process is applied to a benchmark component. An image of the nominal design is presented in Figure 2. 

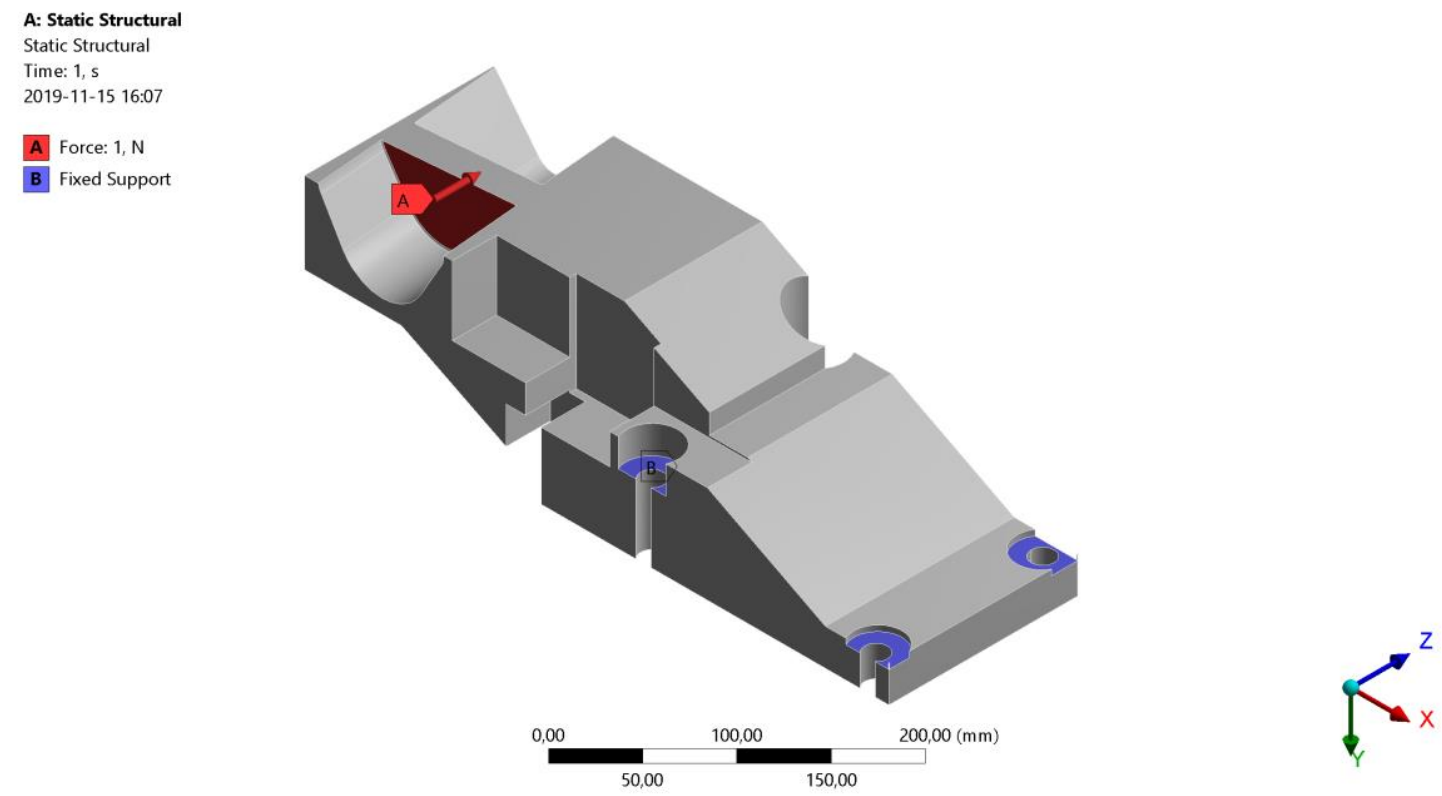

Figure 2. The nominal design space

\subsubsection{Load cases and boundary conditions}

The benchmark component is subject to four load cases. The load cases were created as separate static structural analyses in ANSYS Workbench and applied in the manner represented in Figure 2. The load cases have forces in varying directions and four supports representing a bolt pattern where all degrees of freedom are locked.

\subsection{Machine}

The intended system for manufacturing the part is the 3D Systems DMP 320, which is based on laser powder bed fusion. The modelling and analysis workflow used in this paper is calibrated for this machine, and thus it will be used as the basis for the manufacturing constraints, although the approach presented in this paper is not limited to this machine.

\subsection{Material}

The material selected for the demonstrator component is LaserForm ${ }^{\circledR} 316 \mathrm{~L}$ (A). Mechanical properties from the material supplier can be found in Table 1. Material data from MSC Simufact Material was also used in the later stages of the simulation.

Table 1. Material properties of LaserForm 316L (A) (3D Systems, n.d.)

Measurement

Youngs modulus (GPa)

Ultimate strength (XY) (MPa)

Ultimate strength (MPa) (Z) (MPa)

Yield strength (XY) Rp0.2\% (MPa)

Yield strength (Z) Rp0.2\% (MPa)
Condition

ASTM E8M

$$
180 \pm 15
$$

$660 \pm 20$

$570 \pm 30$

$530 \pm 20$

$440 \pm 20$

\section{Full anneal}

$180 \pm 15$

$610 \pm 30$

$540 \pm 30$

$370 \pm 30$

$320 \pm 20$

\section{Functional design and design for $A M$}

\subsection{Topology optimization}

Several topology optimizations were performed; one optimization without manufacturing constraints and two using an overhang constraint with different build orientations. The results with overhang constraints were compared to the result without the overhang constraint. The build orientation that 
gave the result that most resembled the result without the build orientation constraint was chosen. An image of the results is shown in Figure 3.

No constraint

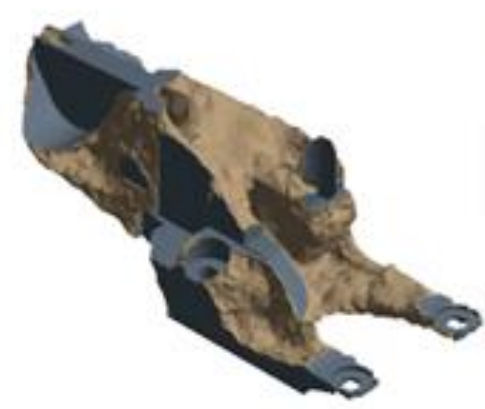

Y direction

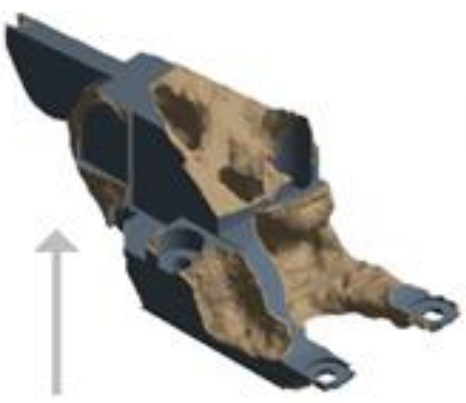

Z direction

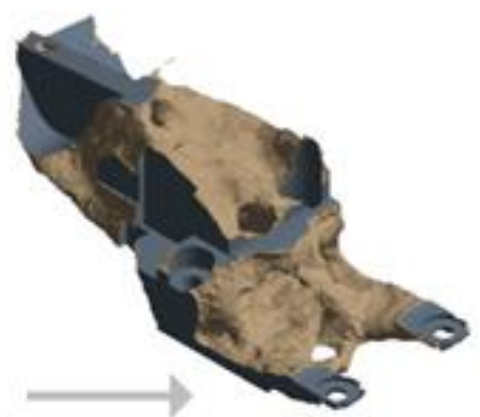

Figure 3. Optimization results without overhang constraints (left) and with overhang constraints in the negative $\mathrm{Y}$ and $\mathrm{Z}$ direction (middle and right)

\subsubsection{Objective}

The objective was to minimize the compliance of the structure.

\subsubsection{Constraints}

All optimizations were performed with a 50\% mass reduction constraint, although other values could be used depending on the target mass and size of the design space. Two of the optimizations were performed with an overhang constraint which minimizes the amount of support material needed for the specified build orientation. Two build orientations were optimized for, $\mathrm{Y}$ and $\mathrm{Z}$.

\subsection{Interpreting the topology optimization results}

The mesh-based result from the topology optimization was imported into the original parametric model and used to trim unnecessary material away and to adapt exterior surfaces to reduce the need for support material. The result is shown in Figure 4. The focus in this step is to create a simple geometry that is efficient to model and which removes the largest areas indicated by the topology optimization. The focus is not to create a model that is identical to the optimization mesh, but rather to reduce the need for support materials, reduce stress concentrators and to remove bulk material. The modelling took around $3 \mathrm{~h}$.

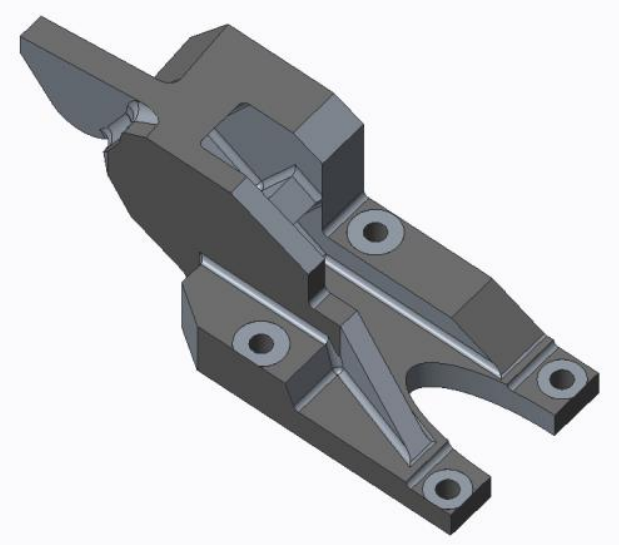

Figure 4. The rough re-design

\subsection{Lattice optimization}

After a new CAD-model has been created based on the initial optimization results, a new optimization is carried out using lattice structures. In the case of the benchmark part, the Octahedral I lattice structure, 
which is one pre-set in ANSYS Mechanical, was used, as this can be built without support structures regardless of lattice size. The lattice size was set to $5 \mathrm{~mm}$, and the minimum and maximum density was set to 0.2 and 0.8 , respectively. This choice was made to avoid very thin struts and very small openings (that could otherwise prevent getting leftover powder out).

In ANSYS Workbench, the current method for obtaining a solid model of the lattice structure is to import the lattice density result (Figure 5) into ANSYS SpaceClaim and to perform a shell operation (Figure 5 and Figure 6). A shell thickness of $2 \mathrm{~mm}$ was chosen.

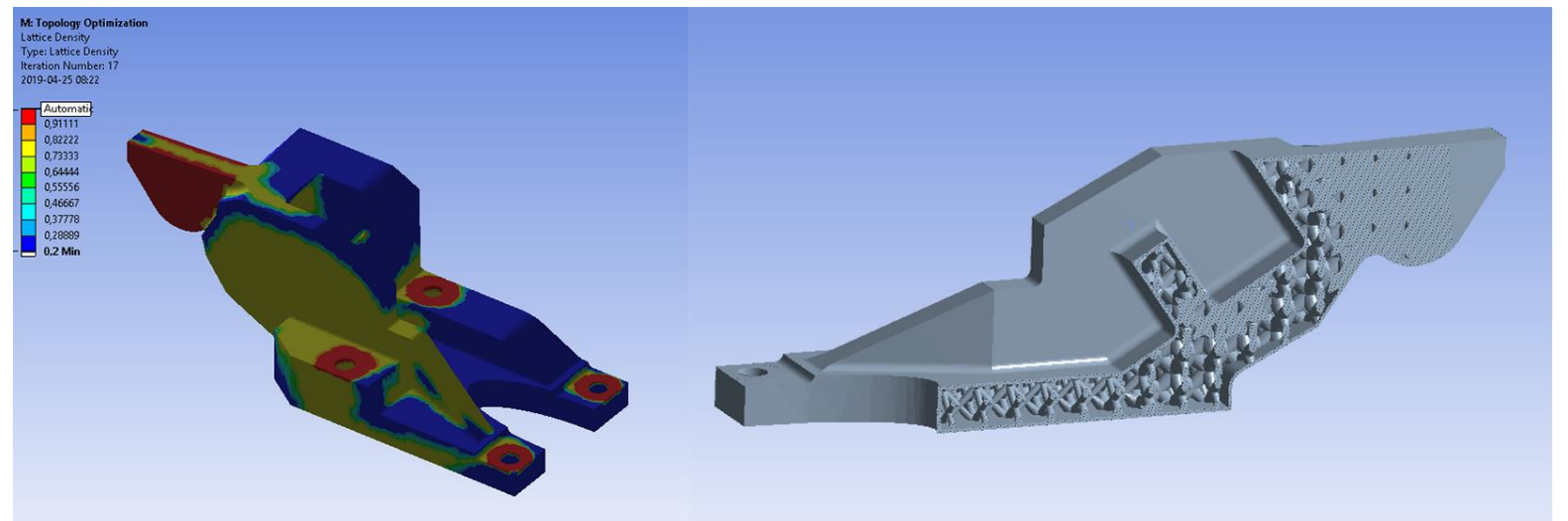

Figure 5. Lattice density (left) and section of the final design with the generated lattice structure (right)

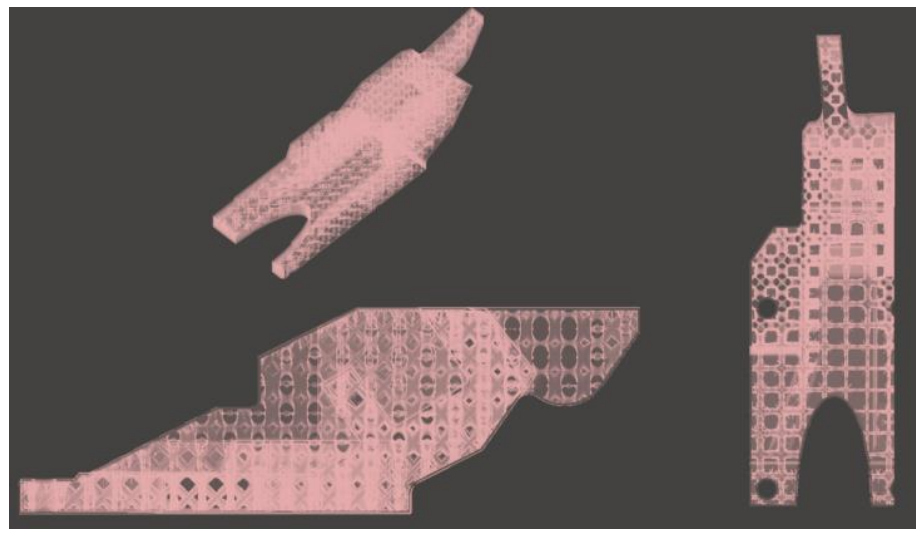

Figure 6. Transparent view of the generated lattice structure

Table 2. Comparison of the volume of the different designs

$\begin{array}{lll}\text { Design } & \text { Volume } & \text { Mass } \\ \text { Nominal } & 4.80 \mathrm{E}-3 \mathrm{~m}^{3} & 38 \mathrm{~kg} \\ \text { Re-design } & 2.9 \mathrm{E}-3 \mathrm{~m}^{3} & 23 \mathrm{~kg} \\ \text { Lattice structure } & 1.4 \mathrm{E}-3 \mathrm{~m}^{3} & 11 \mathrm{~kg}\end{array}$

\subsection{Support design}

The support strategy used in 3D Systems 3DExpert 13 was solid support for the lowest areas, and a wall support for the other areas in need of support. The supports are scanned every second layer (i.e. an effective layer height of $60 \mathrm{um}$ ). The resulting exterior support structure is shown in Figure 7.

As the lattice structure generated by ANSYS SpaceClaim was not enough to support the horizontal internal surfaces, additional, internal solid structures were generated in Rhino and Grasshopper using a custom script. The script generated branching structures that connected the top surfaces of the lattice structures to the bottom surfaces of the outer shell. The resulting structure is shown in Figure 8. This supporting structure is not meant to be removed.

The total weight of the support material is $1.2 \mathrm{~kg}$, or around $10 \%$ of the overall weight. 


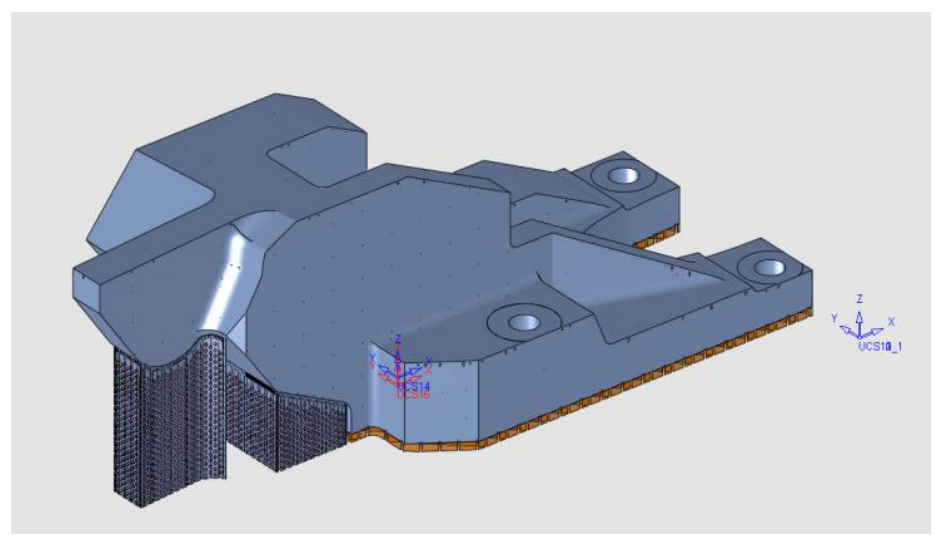

Figure 7. Exterior support structure

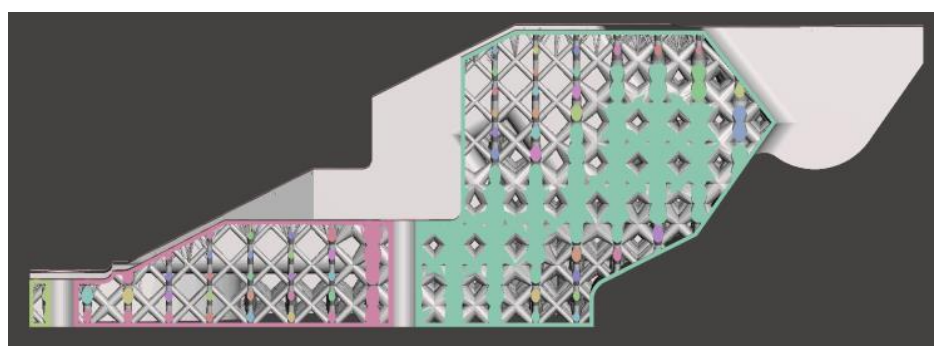

Figure 8. Section showing custom support structure for interior overhangs

\section{Validation}

\subsection{Stress criteria}

The resulting structure from the lattice optimization was imported into a new static structural analysis and meshed using the Body Fitted Cartesian method in ANSYS Workbench with an element size of $2.5 \mathrm{~mm}$. Body Fitted Cartesian was used as it is able to efficiently mesh mesh-based models.

As all parametric information was lost in the conversion from CAD-model to mesh, the loads were applied directly on the finite element mesh. This, in turn, resulted in some singularities due to point loads and point constraints. The stress results and deformations for the most affected load case is shown in Figure 9. The maximum displacement was $0.38 \mathrm{~mm}$. The results are displayed with red showing any area with a stress above the stress constraint. The results indicate that only small areas around the point loads and constraints experienced stresses above the stress constraint.
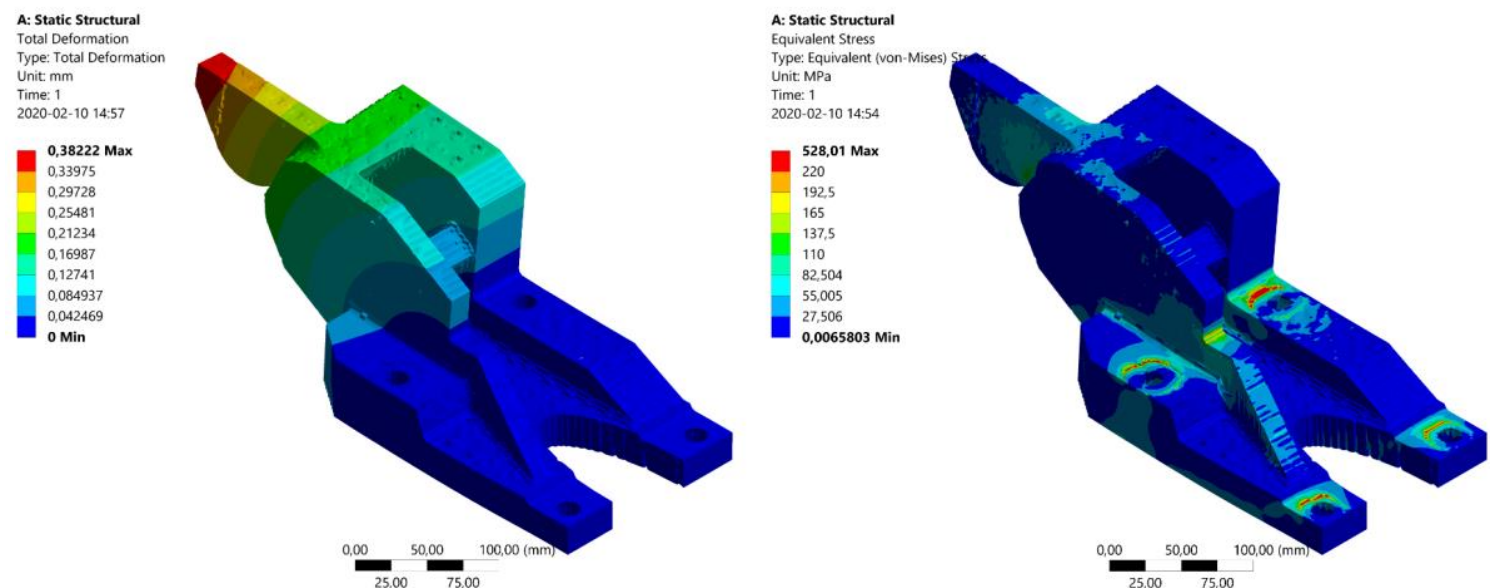

Figure 9. Total deformation (left) and stresses above the stress constraint for the most affected load case (right) 


\subsection{Process simulation}

The process simulation was carried out in MSC Simufact Additive 3.1. The solution method was a mechanical simulation based on calibrated inherent strains, which eliminated the need for a full thermomechanical simulation of the build process. The inherent strains are based on printing a calibration sample in different orientations, and measuring the resulting deformed state of the geometry after support removal. The software will adjust the inherent strains in a virtual model of the calibration geometry, simulate the build and support removal, measure the resulting deformation and compare it to the deformation of the physical sample. If the deformations do not correspond within a given error tolerance, the inherent strains are adjusted and another simulation is run. This process will continue until the error is within the tolerance, or the maximum number of iterations has been reached.

The material was the 316L material supplied as part of the material database Simufact Material 2018. The mesh size used was the same as used for the simulation in ANSYS Workbench, i.e. $2.5 \mathrm{~mm}$. The resulting mesh is shown in Figure 10.

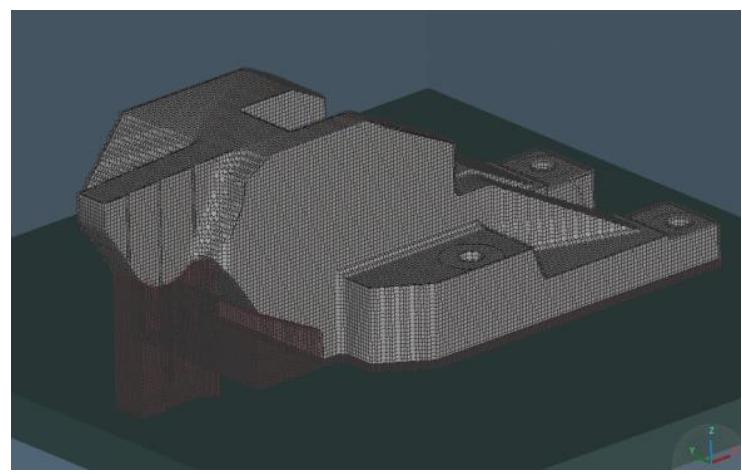

Figure 10. Voxel mesh in Simufact Additive

The results are shown in Figure 11-Figure 12. In summary, the maximum predicted distortion is 1.78 $\mathrm{mm}$, and there is no predicted part failure or re-coater contact during the build. As shown in Figure 12, there are several areas with effective stresses of around 550-600 MPa, which is in line with stresses reported by other sources for SLM-built 316L parts (Wu et al., 2014; Yadroitsev and Yadroitsava, 2015).

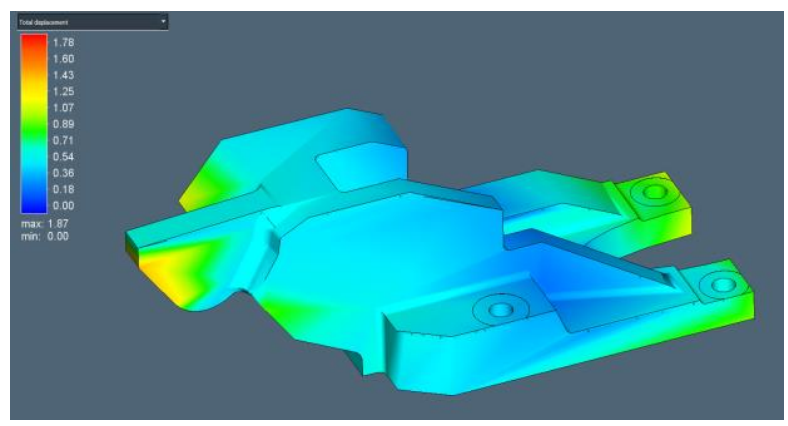

Figure 11. Total displacement after support removal

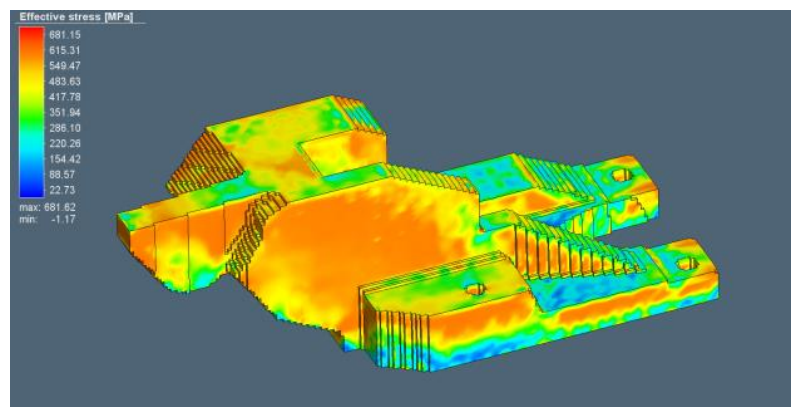

Figure 12. Effective stress in $\mathrm{MPa}$ after support removal 


\section{Comparison to traditional topology optimization}

In order to compare the result of the lattice-based workflow, a more traditional topology optimization was also performed. The goal was to replicate the performance of the lattice-based design, that is, the same or lower stresses, deformation and mass. This was achieved by constraining the mass, stresses and deformation to be lower than the results for the lattice-based design, while minimizing the compliance of the structure. The result is shown in Figure 13. The result clearly indicates that to achieve similar performance to the lattice-based design, substantially more of the outer shell needs to be removed and the load has been entirely concentrated to two screws instead of four. Moreover, interpreting the result and making it manufacturable while maintaining parametric control of the geometry will be challenging and time-consuming.
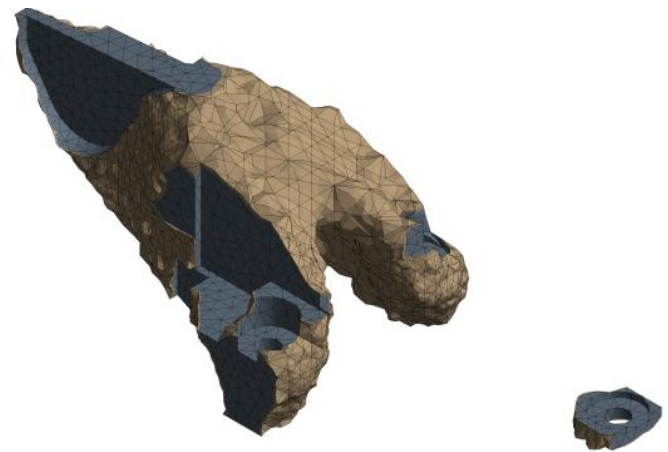

Figure 13. Result of a traditional topology optimization based on the same load cases

\section{Conclusion}

Based on the results presented here, the design fulfils all functional and manufacturing requirements while reducing the volume of the part to just under $30 \%$ of the nominal design. The amount of support material is also low compared to the overall part volume (around 10\%), while being easily removed. With additional design iterations, it is probable that the part volume could be reduced even further. Internal lattice structures have some drawbacks, such as the need for powder removal and the difficulty of post-processing the interior surfaces. Powder removal should not pose an issue with the intended selective laser melting process but the holes in the outer shell need to be carefully placed to avoid highly stressed areas and to promote good flow of the loose powder. If fatigue were to be taken into account, the internal lattice structure would pose post-processing challenges, although some postprocessing techniques based on abrasive fluids could, in theory, generate highly polished surfaces.

The size of the part combined with the lattice structure posed a computational challenge, as the final design with an internal lattice structure was time-consuming to mesh and run simulations on. As such, the stress results presented in Figure 9 and Figure 12 are not necessarily conclusive as a mesh size below $2.5 \mathrm{~mm}$ was unfeasible to solve given the time and resource limitations in this project. Even support generation and Boolean operations proved to be computationally quite time consuming.

\section{Acknowledgements}

This research was conducted as part of the project IDEA-PRINT initiated by RISE (Research Institutes of Sweden) funded by the Swedish Metal Arena for Additive Manufacturing of Metals. Part of this work was done within the project Increasing Excellence on Advanced Additive Manufacturing (INEX-ADAM) funded by the European Union's Horizon 2020 research and innovation programme (GA 810708).

\section{References}

3D Systems (n.d.). "LaserForm 316L (A)", available at: https://www.3dsystems.com/sites/default/files/201706/3D-Systems_LaserForm_316L_\%28A\%29_DATASHEET_A4_US_2017.06.21_WEB.pdf (accessed 13 November 2019). 
Altair (n.d.). "Inspire", available at: https://solidthinking.com/product/inspire/ (accessed 13 November 2019).

ANSYS, I. (n.d.). "Workbench", available at: https://www.ansys.com/products/platform (accessed 13 November 2019).

ANSYS Inc (n.d.). "Topology Optimization Solution Methodology", available at: https://ansyshelp.ansys.com/ account/secured?returnurl=/Views/Secured/corp/v192/wb_sim/ds_topo_solvers.html (accessed 15 November 2019).

“Autodesk Meshmixer” (n.d.). available at: http://www.meshmixer.com/ (accessed 13 November 2019).

Bendsøe, M.P. and Kikuchi, N. (1988), "Generating optimal topologies in structural design using a homogenization method", Computer Methods in Applied Mechanics and Engineering, Vol. 71 No. 2, pp. 197-224.

Bendsøe, M.P. and Sigmund, O. (2004), Topology Optimization: Theory, Methods, and Applications, Springer, Berlin Heidelberg.

Brackett, D., Ashcroft, I. and Hague, R. (2011), "Topology optimization for additive manufacturing", 22nd Annual International Solid Freeform Fabrication Symposium - An Additive Manufacturing Conference, SFF 2011 , pp. 348-362.

Cai, S. et al. (2014), "Stress constrained shape and topology optimization with fixed mesh: A B-spline finite cell method combined with level set function", Computer Methods in Applied Mechanics and Engineering, Vol. 278, pp. 361-387, Elsevier.

Chacón, J.M., Bellido, J.C. and Donoso, A. (2014), "Integration of topology optimized designs into CAD/CAM via an IGES translator", Structural and Multidisciplinary Optimization, Vol. 50 No. 6, pp. 1115-1125, Springer Verlag.

Gaynor, A.T. and Guest, J.K. (2016), "Topology optimization considering overhang constraints: Eliminating sacrificial support material in additive manufacturing through design", Structural and Multidisciplinary Optimization, Vol. 54 No. 5, pp. 1157-1172, Springer Verlag.

Langelaar, M. (2016), "Topology optimization of 3D self-supporting structures for additive manufacturing", Additive Manufacturing, Vol. 12, pp. 60-70.

Liu, J. et al. (2018), "Current and future trends in topology optimization for additive manufacturing", Structural and Multidisciplinary Optimization, Springer Verlag, 1 June.

Liu, J. and Ma, Y. (2016), “A survey of manufacturing oriented topology optimization methods", Advances in Engineering Software, Vol. 100, pp. 161-175, Elsevier Ltd.

Materialise (n.d.). "Magics", available at: https://www.materialise.com/en/software/magics (accessed 13 November 2019).

Mirzendehdel, A.M. and Suresh, K. (2016), "Support structure constrained topology optimization for additive manufacturing", CAD Computer Aided Design, Vol. 81, pp. 1-13, Elsevier Ltd.

Seo, Y.D., Kim, H.J. and Youn, S.K. (2010), "Isogeometric topology optimization using trimmed spline surfaces", Computer Methods in Applied Mechanics and Engineering, Vol. 199 No. 49-52, pp. 3270-3296.

Sigmund, O. and Maute, K. (2013), "Topology optimization approaches", Structural and Multidisciplinary Optimization, Vol. 48 No. 6, pp. 1031-1055, Springer Berlin Heidelberg.

Wang, X. et al. (2016), "Topological design and additive manufacturing of porous metals for bone scaffolds and orthopaedic implants: A review", Biomaterials, Elsevier Ltd, 1 March.

Wu, A.S. et al. (2014), “An Experimental Investigation into Additive Manufacturing-Induced Residual Stresses in 316L Stainless Steel”, Metallurgical and Materials Transactions A: Physical Metallurgy and Materials Science, Vol. 45 No. 13, pp. 6260-6270, Springer Boston.

Yadroitsev, I. and Yadroitsava, I. (2015), "Evaluation of residual stress in stainless steel 316L and Ti6Al4V samples produced by selective laser melting", Virtual and Physical Prototyping, Vol. 10 No. 2, pp. 67-76.

Zegard, T. and Paulino, G.H. (2016), "Bridging topology optimization and additive manufacturing", Structural and Multidisciplinary Optimization, Vol. 53 No. 1, pp. 175-192, Springer Verlag. 